\title{
Minimizing makespan on a single machine with release date and inventory constraints
}

\author{
M. Davari \\ KU Leuven, Research Center for Operations Management (Campus Brussels) \\ e-mail : morteza.davari@kuleuven.be \\ M. Ranjbar \\ Ferdowsi University of Mashhad, Department of industrial Engineering \\ e-mail : m_ranjbar@um.ac.ir \\ R. Leus \\ KU Leuven, Research Group for Operations Research and Business Statistics \\ e-mail : roel.leus@kuleuven.be \\ P. De Causmaecker \\ KU Leuven, Department of Computer Science \\ e-mail : patrick.decausmaecker@kuleuven.be
}

We consider a single machine scheduling problem in which a set of jobs must be processed in a station. Such a station includes a central inventory with a given capacity (limit). The goal is to sequence all jobs in such a way that the inventory and release date constraints are satisfied while the makespan (the completion time of all jobs) is minimized. In this problem, each job is characterized with a processing time, a release date and an inventory modification. A job's inventory modification can be either positive or negative. Jobs with a positive inventory modification increase the central inventory level whereas jobs with negative inventory modification decrease the central inventory level. Obviously the central inventory level must always remain non-negative and not greater than the inventory capacity throughout the scheduling horizon.

The mentioned problem has some interesting applications in truck scheduling at transshipment terminals, known as cross docking in supply chain systems. In a transshipment terminal, trucks arrive in a cross dock either to deliver or to pick up a certain number of goods. Jobs with positive inventory modification imply unloading operations and jobs with negative inventory modification indicate loading operations. Obviously, a loading job can be processed only if the central inventory level is adequately high. Also in the case where the capacity of the inventory is limited, an unloading job can be only be executed if there is still enough space in the inventory.

Inventory constraints have been considered in a number of papers in the field of scheduling. However, there are a very limited number of papers considering machine scheduling subject to inventory constraints. We cite Briskorn and Leung [1] who show that a single machine scheduling problem with inventory constraints to 


\begin{tabular}{llllll}
\hline Method & \multicolumn{5}{c}{$n$} \\
\cline { 2 - 6 } & 10 & 20 & 30 & 40 & 50 \\
\hline DP & 0.00 & 0.11 & 373.24 & $(96)$ & $(96)$ \\
BB & 0.00 & 1.94 & $(17)$ & $(26)$ & $(34)$ \\
\hline
\end{tabular}

TABLE 1 - Average CPU times (in seconds) or number of unsolved instances within the time limit (out of 96) for different values of $n=10,20,30,40$ and 50.

minimize the maximum lateness is NP-hard. They also develop a set of branchand-bound algorithms that solve instances of the mentioned problem. Unlike Briskorn and Leung [1] who consider single machine scheduling problems, Bazgosha et al. [2] consider a transshipment terminal with a set of parallel stations for loading and unloading operations. They consider this problem as a parallel machine scheduling problem with release dates and inventory constraints.

Recently, Ghorbanzadeh et al. [3] proposed a branch-and-bound algorithm and dynamic programming algorithm to solve the problem studied in this paper. Alternatively, we propose a totally novel scheduling terminology and an advanced solution approach, namely a block-based branch-and-bound algorithm, to solve the instances of our problem efficiently. The contributions of this paper are fourfold :

1. we prove that the introduced problem is NP-hard in the strong sense by a reduction from 3 -Partition;

2. we introduce the concept of block-based scheduling with which we propose a novel problem formulation;

3. we provide some theoretical results for instances with certain structure, based on which we classify instance into two groups of 'easy' and 'hard' instances; and

4. we propose two MILP formulations and a block-based branch-and-bound algorithm to solve the problem until optimality.

We compare our proposed branch-and-bound (BB) algorithm with the dynamic programming approach (DP) (from [3]) and report some preliminary results in Table 1. These results suggest that although DP performs better for small instances, BB is better at solving large instances within the time limit (which is 1000 seconds) than DP.

\section{Références}

[1] Briskorn, D. \& Leung, J. Y.-T. Minimizing maximum lateness of jobs in inventory constrained scheduling. Journal of the Operational Research Society, 2013, 64, 1851-1864

[2] Bazgosha, A.; Ranjbar, M. \& Jamili, N. Scheduling of loading and unloading operations in a multi stations transshipment terminal with release date and inventory constraints. Computers $\&$ Industrial Engineering, 2017, 106, 20-31

[3] Ghorbanzadeh, M. ; Ranjbar, M. \& Jamili, N. Transshipment scheduling at a single station with release date and inventory constraints. Ferdowsi University, 2017 